\title{
Optimization analysis of high-speed container flat car suspension parameters
}

\author{
Menggang Guo ${ }^{1, a}$, Pingbo $\mathrm{Wu}^{2, \mathrm{~b}}$ and Ren $\mathrm{Luo}^{2, \mathrm{c}}$ \\ ${ }^{1}$ School of southwest jiaotong University, Chengdu 610031, China; \\ ${ }^{2}$ School of southwest jiaotong University, Chengdu 610031, China. \\ amenggangcool@163.com, 'bupingbo@163.com, '149162368@qq.com
}

Keywords: High-speed wagon, bogie, parameters of suspension, equivalent conicity, stability

\begin{abstract}
Transportation has a rapid development with the stimulus of electronic commerce. As the internet economic hold dominant position, freight characterized by mass high added value, strong timeliness demand has been in strong demand. The world railway transportation presents the trend of logistics. Based on the freight market demand, high-speed wagon become one of the main direction of railway transportation. The technique of railway freight has restricted the reform of the railway freight in our country. The high speed bogie container flat car of Speed of $220 \mathrm{~km}$ has been designed. This article will optimize its key parameters of suspension.
\end{abstract}

\section{The research background}

According the bogie and car body's parameters of container flat car of Speed of 220 designed by one company, referring to the high-speed motor train unit's bogie structure, we adopt the method of dynamic simulation to analyze the motion stability in flat car empty condition.

\section{Introduction of bogie}

The high-speed bogie is mainly composed of frame, axle box, rotary arm, wheel, traction, braking system, etc. The primary positioning structure adopts the axle box tumbler, and installs a vertical steel spring and hydraulic damper. Second suspension system adopt rubber spring including secondary lateral dampers, yaw damper, secondary lateral rubber stop, traction rod device, etc. The Fig.1 shows the structure of bogie.

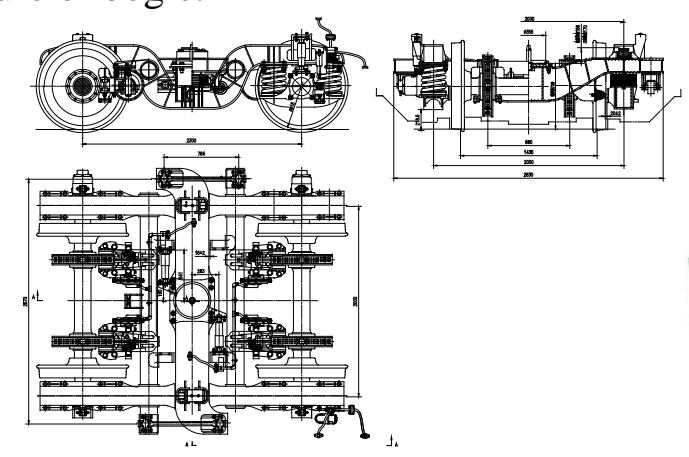

Fig.1 structure of bogie.

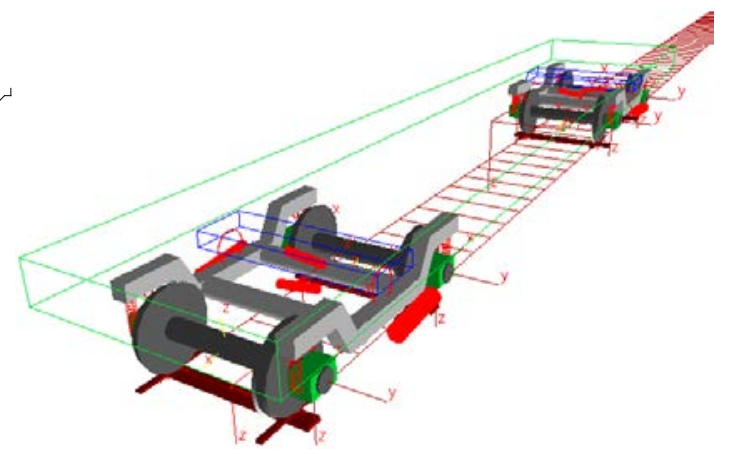

Fig.2 dynamic modal.

\section{Analysis method}

\subsection{Analysis method of stability}

This paper first established a simulation model of vehicle dynamic as shown in Fig.2. Vehicle system matrix is calculated by using the linearization method, while getting its eigenvalue and eigenvector. Eigenvalue correspond to the damp ratio and frequency of the vehicle system motion modes, and eigenvector correspond to its motion modes [1]. We can get the damping ratio by numerical calculation, when minimum damping ratio is more than $5 \%$, we consider the system is stable, otherwise the system is unstable [2]. 
$5 \%$ is thought to be the minimum damping ratio to maintain stability of the mechanical system. With the increase of Equivalent conicity, damping ratio increase first then diminish. In the equivalent conicity is relatively small, when the operating speed is relatively high, hunting may occur body; when the equivalent conicity relatively large, may occur bogie hunting. Fig. 3 shows the rule.

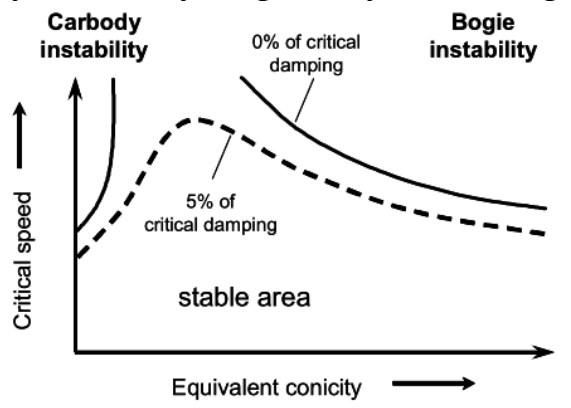

Fig.3 The modal damping ratio to the motion stability.

\subsection{Relationship between wheel and rail.}

Depending on the wheel and rail profile appearance, as well as wheel-rail geometry parameters, we can calculate accurately wheel track relationship. This relationship includes the information of the wheel-rail contact point, the contact angle, contact radius, etc. Quasi-linear model parameter of wheel-rail contact is calculated by harmonic linearization, which we consider this linearization is applicable to small wheel displacements near nominal contact, and wheel displacements at the wheel-rail clearance. [3] By linear analysis, we only can use a specific parameter value of equivalent conicity (lamd), contact angle (eps) and roll angle (sig). And there is a certain percentage of their relationship. Sig $=0.2$ lamd, Eps $=85$ lamd [4].

\section{Impact of vehicle suspension parameters}

The primary suspension adopt the tumbler, and install a vertical steel spring and hydraulic damper. Secondary suspension use air spring structure, and install yaw dampers, secondary lateral damper; We will analyze the influence of its parameters on the motion stability while the equivalent conicity's value is $0.10 、 0.15 、 0.20 、 0.30$ and 0.40 .

\begin{tabular}{cccc}
\multicolumn{4}{c}{ Main performance parameters } \\
\hline axil load & $18 \mathrm{t}$ & weight & $27 \mathrm{t}$ \\
\hline $\begin{array}{c}\text { Distance betwee } \\
\mathrm{n} \text { bogie centers }\end{array}$ & $10.5 \mathrm{~m}$ & designed for speed & $220 \mathrm{~km} / \mathrm{h}$ \\
\hline track gauge & $1435 \mathrm{~mm}$ & $\begin{array}{c}\text { Minimum curve } \\
\text { radius }\end{array}$ & $145 \mathrm{~m}$ \\
\hline
\end{tabular}

\subsection{The primary longitudinal positioning stiffness.}

The primary longitudinal stiffness is an important factor of stability. One hand when the stiffness is too small, it will lead to the lack of ability to locate and low critical speed. On the other hand, when the stiffness is so big that the bogie will be closer to the rigid bogie, and it leads to the poor stability. To optimize the high speed train of primary longitudinal stiffness, we need combine wheel-rail contact relationship. [5]

From the simulation results as shown in Fig.4 which the lower left is stable and unstable at the upper right. In order to satisfy a certain stability, the primary longitudinal stiffness need to be smaller with the equivalent conicity smaller. When the primary longitudinal stiffness is bigger than $30 \mathrm{MN} / \mathrm{m}$, the bigger equivalent conicity is, the higher critical speed will be. At the same level of equivalent conicity, critical speed will decreases with increasing stiffness. Under the comprehensive comparison, when the primary longitudinal stiffness around $20 \mathrm{MN} / \mathrm{m}$, the stability will be relatively good. 


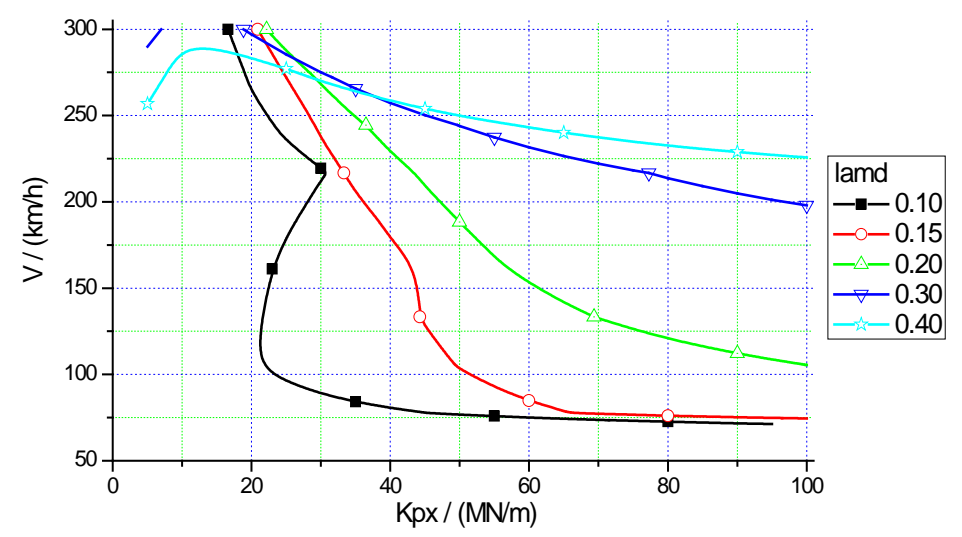

Fig.4 Change of stability in different equivalent conicity and different longitudinal stiffness.

\subsection{Yaw damper to stability}

The actual hydraulic damper has a rubber joints both ends. These make hydraulic damper appears elastic force in the axial direction and show a certain stiffness effect. When considering the hydraulic shock absorber rubber joint stiffness and the stiffness of liquid, it will produce a phase change between the damping force and the piston speed. This feature is called dynamic damping characteristics. [6, 7] Indeed, it should be seen as a combination element of damper and spring, which called Maxwell Model.

\subsubsection{Equivalent series dynamic stiffness of yaw damper}

Under conditions of the basic parameters, the calculation results can be seen in the Fig5. When the equivalent taper 0.1, yaw damper stiffness greatest impact on the stability, In this case, the stability are deficient under a variety of equivalent stiffness; When the equivalent conicity is greater than 0.15 , the steady speed increased with yaw damper equivalent stiffness, reduced after the first increase. And also the optimal value increases with equivalent conicity. Optimal values range 4MN / $\mathrm{m}$ to between $8 \mathrm{MN} / \mathrm{m}$.

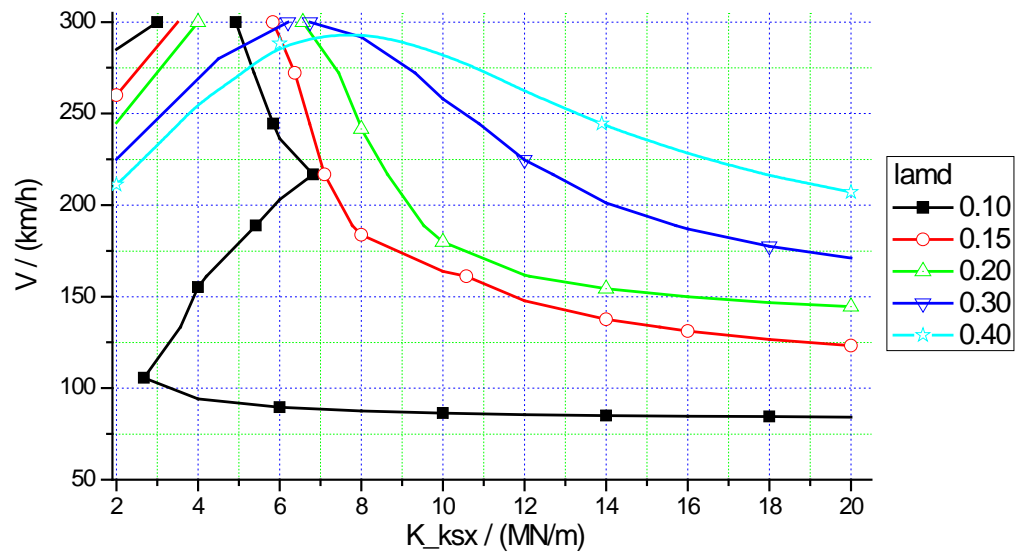

Fig.5 Change of stability in different equivalent conicity and different stiffness of yaw damper.

\subsubsection{Equivalent series dynamic damping of yaw damper.}

According to the previous optimization, equivalent series dynamic stiffness of yaw damper takes the value of $6.0 \mathrm{MN} / \mathrm{m}$. we will analysis the influence of Maxwell model equivalent series damping of yaw damper and different equivalent conicity.

Under conditions of the basic parameters, the result is shown in Fig.6. We can see when the equivalent conicity is 0.1 , yaw damper damping influence on the stability is more significant. Equivalent damping value requires more than $1100 \mathrm{kN}$.s / m, the vehicle stability of the system will meet the requirements. When the equivalent conicity is greater than 0.15 , critical speed shows little change. Equivalent damping value requires more than $200 \mathrm{kN} . \mathrm{s} / \mathrm{m}$, we wil get better vehicle stability systems. When the equivalent conicity is relatively large, equivalent damping yaw damper is not the bigger the better. 


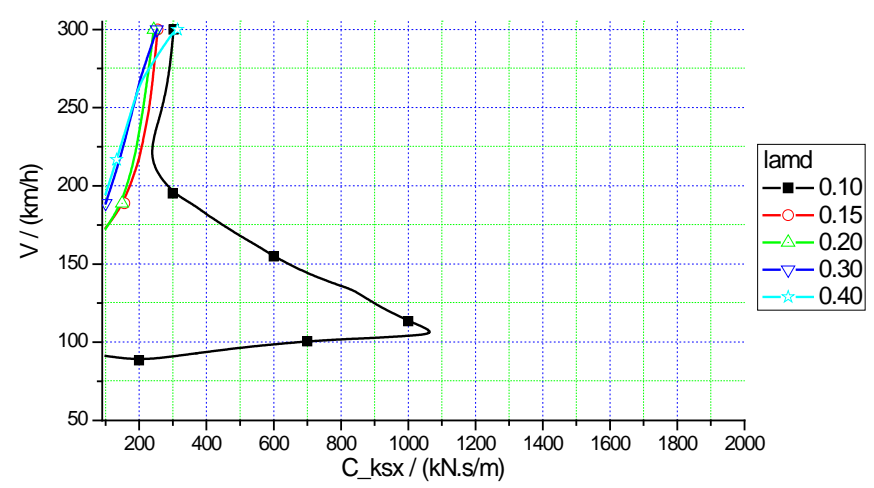

Fig.6 Change of stability in different equivalent conicity and different damping of yaw damper.

\section{Summary}

According to the given $220 \mathrm{~km} / \mathrm{h}$ High Speed container flat car which length between Truck centers is $10.5 \mathrm{~m}$, we analyzed the influence of main suspension parameters on the stability of motion under empty state. Because the shorter length between Truck centers, the mass and inertia is relatively small, vehicle system has poor motion stability, and is more sensitive to parameters.

1. In order to satisfy a certain stability, the primary longitudinal stiffness need to be smaller with the equivalent conicity smaller.

2.When the equivalent taper 0.1 , both the stiffness and the damping of yaw damper greatest impact on the stability.

3 .When the equivalent conicity is relatively large, equivalent damping yaw damper is not the bigger the better.

\section{Acknowledgment}

This work has been supported by the China Railway Corporation scientific research and development projects (project No. 2014J008-A and project No. 2014J008-B) and the Chinese National Science Foundation (project No. 51475388).

\section{References}

[1]. Futian Wang. Vehicle System Dynamics. China Railway Publishing House. 1994-08, p.59-77.

[2]. Weihua Zhang. High Speed Train Design Method of Motion Stability. Journal of Southwest Jiaotong University .Vol.48 (2013) No. 01,p.1-9.

[3]. Fengtao Lin. Comparison of two quasi-linear calculation method of equivalent conicity. Railway Locomotive \&Car. Vol.33 (2013) No.01,p.53-58

[4]. EN15302 Railway applications, Method for Determining the Equivalent conicity[s].CEN, Brussels, 2008.

[5]. Ren Luo. High-speed emu line running adaptability Journal of Traffic and Transportation Engineering .2011(06):37-43.

[6]. Guozhen Yang, Wangfutian Locomotive Hydraulic Damper[M] China Railway Publishing House.2002.

[7]. EN 13802 Railway Applications - Suspension Components - Hydraulic Dampers.2014. 